\title{
Efficiency of individual dosage of digoxin with calculated concentration
}

This article was published in the following Dove Press journal:

Clinical Interventions in Aging

22 July 2014

Number of times this article has been viewed

\author{
Li Zhao',* \\ Peng Yang $2, *$ \\ Pengmei $\mathrm{Li}^{\prime}$ \\ Xiaoxing Wang' \\ Wangjun Qin' \\ Xianglin Zhang' \\ 'Department of Pharmacy, \\ ${ }^{2}$ Department of Cardiology, \\ China-Japan Friendship Hospital, \\ Beijing, People's Republic of China \\ *These authors contributed equally \\ to this work
}

Background: Digoxin is a frequently prescribed drug, particularly in the elderly population, in which there is an increased prevalence of atrial fibrillation and cardiac failure. With its complex pharmacokinetic profile and narrow therapeutic index, use of digoxin requires regular monitoring of blood levels. Recent evidence suggests that a lower concentration range $(0.4-1.0 \mathrm{ng} / \mathrm{mL})$ is preferable in patients with congestive heart failure and a higher range $(0.8-2.0 \mathrm{ng} / \mathrm{mL})$ is needed in patients with atrial tachyarrhythmia. The Konishi equation is widely used to predict the serum digoxin concentration (SDC) in Japan. This study assessed the correlation between SDC predicted by the Konishi equation and that actually measured in Chinese patients and investigated the impact of renal function on SDC.

Methods: The study subjects comprised 72 patients with cardiac failure or/and atrial tachyarrhythmia seen at our hospital from January 2012 to December 2013. The patients were divided into five groups according to Kidney Diseases Outcome Quality Initiative guidelines. SDCs were measured using the Abbott Architect 11000 immunology analyzer. The correlations between measured SDCs and calculated SDCs and between clearance of digoxin and creatinine clearance rate were assessed retrospectively.

Results: The correlation between measured and predicted SDC calculated by the Konishi equation was significant $(r=0.655, P<0.001$ ) for the 72 patients overall; however, correlations within the different stages of renal function were nonsignificant, with a correlation found only in patients with stage 3 ( $30 \mathrm{~mL}$ per minute $<$ creatinine clearance $<60 \mathrm{~mL}$ per minute). With regard to the correlation between clearance of digoxin and creatinine clearance, our results show that although there was a significant correlation between clearance of digoxin and creatinine clearance in the group overall, correlations were not evident within the different stages of renal function.

Conclusion: The results of this study indicate that clearance of digoxin and the creatinine clearance rate cannot be explained by renal function alone and that the validity of the Konishi equation for individualizing the digoxin dosage in Chinese patients is limited, being applicable only in stage 3 renal disease. Further research in larger numbers of patients across all stages of renal function will be required in the future to verify the original Konishi model.

Keywords: serum digoxin concentration, predicted concentration, renal insufficiency

\section{Introduction}

Digoxin, a cardiac glycoside, is the oldest known positive inotropic agent and has been used for approximately 200 years in the treatment of heart failure and atrial tachyarrhythmia. The disadvantages of digoxin are its narrow therapeutic index and complex pharmacokinetics in different individuals. The frail elderly with comorbidities, who are often being treated with a number of other medicines, are at particular risk of digoxin toxicity. ${ }^{1}$ With the development of analytical methods, therapeutic drug monitoring has become an integral part of digoxin prescribing, ${ }^{2}$ allowing medical practitioners to optimize therapy while significantly reducing the incidence of toxicity. ${ }^{3}$ Recent evidence suggests that a lower range $(0.4-1.0 \mathrm{ng} / \mathrm{mL})$ is preferable for patients with 
congestive cardiac failure and a higher range $(0.8-2.0 \mathrm{ng} / \mathrm{mL})$ is suitable for patients with atrial tachyarrhythmia. ${ }^{4}$ Measurement of serum digoxin concentration (SDC) can contribute to the clinical diagnosis. Close monitoring of changes in SDC and in particular calculation of the target dose are important. A number of equations have been developed to calculate the digoxin dosage in order to avoid the adverse effects of overexposure to the drug, but most have targeted higher SDCs than those now recommended due to safety concerns. Konishi ${ }^{5}$ formulated an equation to determine the daily dose of digoxin based only on creatinine clearance rate (Ccr) and SDC. However, this equation has not been tested in Chinese patients. Therefore, the suitability of the equation for the Chinese population has not yet been assessed.

The aim of this study was to evaluate the correlation between SDC predicted by the Konishi equation, ${ }^{5}$ ie, $\mathrm{L}(\mathrm{ng} / \mathrm{mL})=\mathrm{D}(\mu \mathrm{g} /$ day $) /[2.22 * \mathrm{Ccr}+25.7]$, and the actual SDC measured in Chinese patients at different stages of renal disease, with Ccr values estimated by the Cockcroft-Gault equation in $\mathrm{mL}$ per minute. ${ }^{6}$

\section{Materials and methods}

\section{Study design and patient population}

This was a descriptive retrospective study conducted at the China-Japan Friendship Hospital, Beijing, People's Republic of China, between January 2012 and December 2013. Inclusion criteria were symptomatic heart failure (New York Heart Association functional class $\leq 2$ ) and left ventricular systolic dysfunction $\leq 45 \%$ or atrial tachyarrhythmia, and age $\geq 18$ years. Exclusion criteria were mainly related to absolute or relative contraindications to digoxin, including grade II-III atrioventricular block without a pacemaker, sinus bradycardia (resting heart rate $<50$ beats per minute) without a pacemaker, hypokalemia $(<3.5 \mathrm{mmol} / \mathrm{L})$, hypercalcemia $(>2.64 \mathrm{mmol} / \mathrm{L})$, and hypertrophic cardiomyopathy. The following clinical and biochemical data were recorded for 72 patients: age, sex, Ccr, risk factors (eg, diabetes, hypertension, hypercholesterolemia, smoking), and concomitant medications (Table 1). The patients were classified into stages $1-5$ according to Kidney Diseases Outcome Quality Initiative guidelines ${ }^{7}$ (Table 2).

\section{Time of sampling and the Konishi equation}

All patients received digoxin tablets orally at a dosage of $0.125 \mathrm{mg}$ or $0.25 \mathrm{mg}$ every $24-48$ hours after being admitted to hospital. Serum digoxin samples were drawn 24-48 hours before the next dose after steady-state digoxin concentrations had been achieved. SDCs were measured using the chemiluminescence microparticle immunoassay (Architect i1000, Abbott GmbH, Wiesbaden, Germany). A correlational analysis of measured SDCs and calculated SDCs was then performed.

We calculated the predicted concentration of digoxin using the Konishi equation, as follows:

$$
\mathrm{L}(\mathrm{ng} / \mathrm{mL})=\mathrm{D}(\mu \mathrm{g} / \text { day }) /[2.22 * \mathrm{Ccr}+25.7]
$$

where $\mathrm{L}$ is the serum digoxin concentration and $\mathrm{D}$ is the dose of digoxin. Ccr was estimated by the Cockcroft-Gault equation, as follows:

$\operatorname{Ccr}(\mathrm{mL}$ per minute $)=(140-$ age $) *($ weight in $\mathrm{kg})$ $*(0.85$ if female $) /(72 * \mathrm{Cr}[\mathrm{mg} / \mathrm{dL}])(2)$

In order to investigate whether renal function would affect the clearance of digoxin, the following conventional pharmacokinetic equation was used:

$$
\text { Clearance }(\mathrm{CL})=(\text { dosage } / \text { dosage interval }) / \text { Css }
$$

in which Css was SDC at steady state. Although the Css is higher than the digoxin trough concentration, the ratio of Css to the trough concentration is presumed to be constant. ${ }^{8}$ Therefore, the above equation can be given as:

$$
\mathrm{CL}=\alpha \times(\text { dosage } / \text { dosage interval }) / \mathrm{L}
$$

where $\mathrm{L}$ is the digoxin trough concentration and $\alpha$ is a constant.

\section{Statistical analysis}

Continuous variables are shown as the mean \pm standard deviation and were compared using the Student's $t$-test or one-way analysis of variance as appropriate. Categorical data are expressed as numbers. Significant differences between variables were demonstrated by one-way analysis of variance and post hoc comparisons between the five stages of renal function were performed using Tamhane's T2 test for multiple comparisons. The correlation between measured and predicted SDCs was tested with a linear regression model. All statistical analyses were performed using Statistical Package for the Social Sciences version 19.0 software (IBM Corporation, Armonk, NY, USA), with a two-tailed probability value of $<0.05$ considered to be statistically significant.

\section{Results}

The baseline characteristics of the 72 patients in this study are shown in Table 1. The mean patient age was $71.5 \pm 13.0$ years and the mean estimated Ccr was less than $49.5 \pm 31.1 \mathrm{~mL}$ 
Table I Baseline characteristics of patients

\begin{tabular}{ll}
\hline Variables & Total $(\mathbf{n}=\mathbf{7 2})$ \\
\hline Age, years, mean \pm SD & $71.5 \pm 13.0$ \\
Male, $\mathrm{n}(\%)$ & $55.6 \%$ \\
Ccr, $\mathrm{mL}$ per minute, mean \pm SD & $49.5 \pm 31 . \mathrm{I}$ \\
Risk factors, $\mathrm{n}(\%)$ & \\
DM & $15(20.8 \%)$ \\
Hypertension & $26(36.1 \%)$ \\
Hypercholesterolemia & $12(16.7 \%)$ \\
Smoking & $9(12.5 \%)$ \\
Concomitant medications, $\mathrm{n}(\%)$ & \\
Amiodarone & $2(2.8 \%)$ \\
CCBs & $1(1.4 \%)$ \\
Spirolactone & $27(37.5 \%)$ \\
Furosemide & $15(20.8 \%)$ \\
ACEls & $0(0 \%)$ \\
Antifungal agents & $0(0 \%)$ \\
\hline
\end{tabular}

Abbreviations: SD, standard deviation; $C c r$, creatinine clearance rate; $D M$, diabetes mellitus; CCB, calcium blocker; ACEl, angiotensin-converting enzyme inhibitor.

per minute. SDC according to digoxin dosage and renal function is shown in Table 2. Overall, only 56 measured SDCs $(77.8 \%)$ were within the defined therapeutic range $(0.4-2.0 \mathrm{ng} / \mathrm{mL})$, seven $(9.7 \%)$ were in the subtherapeutic range $(<0.4 \mathrm{ng} / \mathrm{mL})$, and nine $(12.5 \%)$ were in the supratherapeutic range $(>2.0 \mathrm{ng} / \mathrm{mL})$.

By logarithmic transformation, all data for measured concentrations fitted a normal distribution. Figure 1 shows the measured SDCs for patients according to stage of renal disease. SDCs for stage 1 patients were significantly lower than for patients in the other four stages $(P<0.01$, analysis of variance), and SDCs in stage 2 patients were lower than those in patients with stage 4 or stage 5 disease $(P<0.01$, analysis of variance). However, there were no significant differences between any other groups. The nine patients within the supratherapeutic range had a mean age exceeding 65 years and were found to have severe renal dysfunction, indicating that digoxin can accumulate more easily in the body and cause toxicity in elderly patients with decreased renal function.

The correlation between the measured SDC and the SDC predicted by the Konishi equation was significant $(r=0.655$, $P<0.001$, Figure 2) for the 72 patients overall, while the correlations within the different stages of renal function were nonsignificant, except for a correlation found in patients with Stage 3. Our results show that the correlation was significant in patients with stage $3(30 \mathrm{~mL}$ per minute $<\mathrm{Ccr}<60 \mathrm{~mL}$ per minute) but not in patients with other stages. Although there was a significant correlation between $\mathrm{CL}$ and $\mathrm{Ccr}$ in the group overall, correlations were not evident within the different stages of renal function (Figure 3). The CL of digoxin could not be explained by renal function alone, and the validity of the Konishi equation for individualizing the digoxin dosage in Chinese patients was only applicable in stage 3 patients.

\section{Discussion}

Although digoxin has been used for over two centuries, concern about its toxicity can be traced back to its introduction into therapy. However, considering its beneficial effect in reducing mortality and hospitalization in patients with progressive heart failure, its cost-effectiveness, and easy availability worldwide, digoxin should not be considered as a drug of the past but rather a drug of the present and even one of the future. ${ }^{9}$ In this study, nine patients with supratherapeutic digoxin concentrations were found to have severe renal dysfunction, and their mean age exceeded 65 years. It has been demonstrated that digoxin can accumulate in the body easily and cause toxicity in elderly patients with decreased renal function. ${ }^{9}$ Further, it should be noted that elderly patients with renal dysfunction are particularly susceptible to developing

Table 2 SDC in different digoxin dosage or renal function groups

\begin{tabular}{|c|c|c|c|c|}
\hline & \multicolumn{3}{|c|}{ SDC (ng/mL) } & \multirow[t]{2}{*}{ Total } \\
\hline & $<0.4$ & $0.4-2.0$ & $>\mathbf{2 . 0}$ & \\
\hline \multicolumn{5}{|l|}{ Digoxin dosage (mg/day) } \\
\hline 0.625 & I (I.4\%) & $7(9.7 \%)$ & $2(2.8 \%)$ & $10(13.9 \%)$ \\
\hline 1.25 & $6(8.3 \%)$ & $46(63.9 \%)$ & $2(2.8 \%)$ & $54(75 \%)$ \\
\hline 2.50 & $0(0 \%)$ & $3(4.2 \%)$ & $5(6.9 \%)$ & 8 (II.1\%) \\
\hline$\%$ within dosage group & $7(9.7 \%)$ & $56(77.8 \%)$ & $9(12.5 \%)$ & $72(100 \%)$ \\
\hline \multicolumn{5}{|c|}{ Stages: Ccr (mL per minute) } \\
\hline Stage I, $>90$ & I (I.4\%) & $7(9.7 \%)$ & $0(0 \%)$ & $8(11.1 \%)$ \\
\hline Stage 2, 60-90 & $4(5.6 \%)$ & $12(16.7 \%)$ & I (I.4\%) & $17(23.6 \%)$ \\
\hline Stage $3,30-60$ & $2(2.8 \%)$ & $23(31.9 \%)$ & $2(2.8 \%)$ & $27(37.5 \%)$ \\
\hline Stage $4,15-30$ & $0(0 \%)$ & $10(13.9 \%)$ & $3(4.2 \%)$ & $13(18.1 \%)$ \\
\hline Stage $5,<15$ & $0(0 \%)$ & $4(5.6 \%)$ & $3(4.2 \%)$ & $7(9.7 \%)$ \\
\hline$\%$ within Ccr group & $7(9.7 \%)$ & $56(77.8 \%)$ & $9(12.5 \%)$ & $100 \%$ \\
\hline
\end{tabular}

Note: Data show the number of patients (\% of total).

Abbreviations: $\mathrm{Ccr}$, creatinine clearance rate; SDC, serum digoxin concentration. 


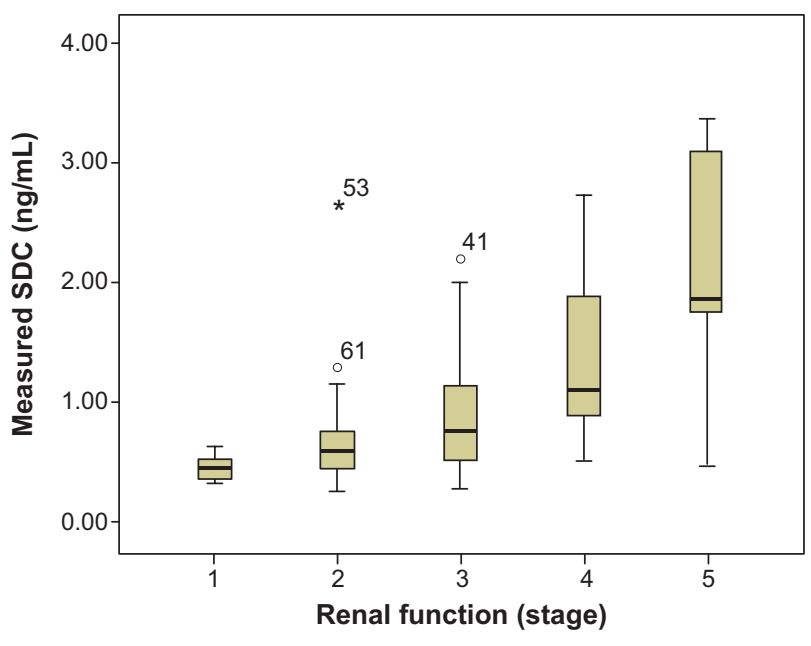

Figure I Differences in SDC in patients with different levels of renal function. Note: ${ }^{\circ}$ Represents a mild outlier and *an extreme outlier.

Abbreviation: SDC, serum digoxin concentration.

high concentrations of digoxin, with an increased risk of cardiac toxicity, which consequently increases the mortality risk. ${ }^{10}$ The 2013 American College of Cardiology Foundation/ American Heart Association guidelines recommend using a lower digoxin dosage of $0.125 \mathrm{mg}$ daily or $0.125 \mathrm{mg}$ every other day in patients older than 70 years or with impaired renal function. ${ }^{11}$

Digoxin is eliminated $50 \%-70 \%$ unchanged via the kidneys, with the remainder being metabolized or excreted via the biliary route. ${ }^{12}$ Assuming no clinically significant interindividual difference in nonrenal digoxin CL owing to the lack of a compensatory increase in metabolic clearance with a decrease in the renal clearance, Konishi developed a predictive model in order to apply the equation in clinical practice for accurate and rapid determination of digoxin concentration. ${ }^{5}$ However, in subsequent research, it was found that only $26 \%$ of interindividual variability in digoxin CL can be explained by changes in Ccr. ${ }^{13}$ Although a study reported by Muzzarelli et a $1^{14}$ also supported the clinical validity of the Konishi equation for calculation of the individual digoxin dosage for Caucasians, the exclusion criteria in their study included severe renal insufficiency ( $\mathrm{Crr}<30 \mathrm{~mL}$ per minute) and their study population was small, which may cause deviation of the results.

Our study shows that although the correlation between the measured SDC and that predicted by the Konishi equation was significant for the 72 patients overall $(r=0.655, P<0.001$, Figure 2), no correlation was found within the different stages of renal function except for stage 3 patients. It was also shown that although there was a significant correlation between $\mathrm{CL}$ and $\mathrm{Ccr}$ in the group overall, correlations within the different stages of renal function were not evident (Figure 3).
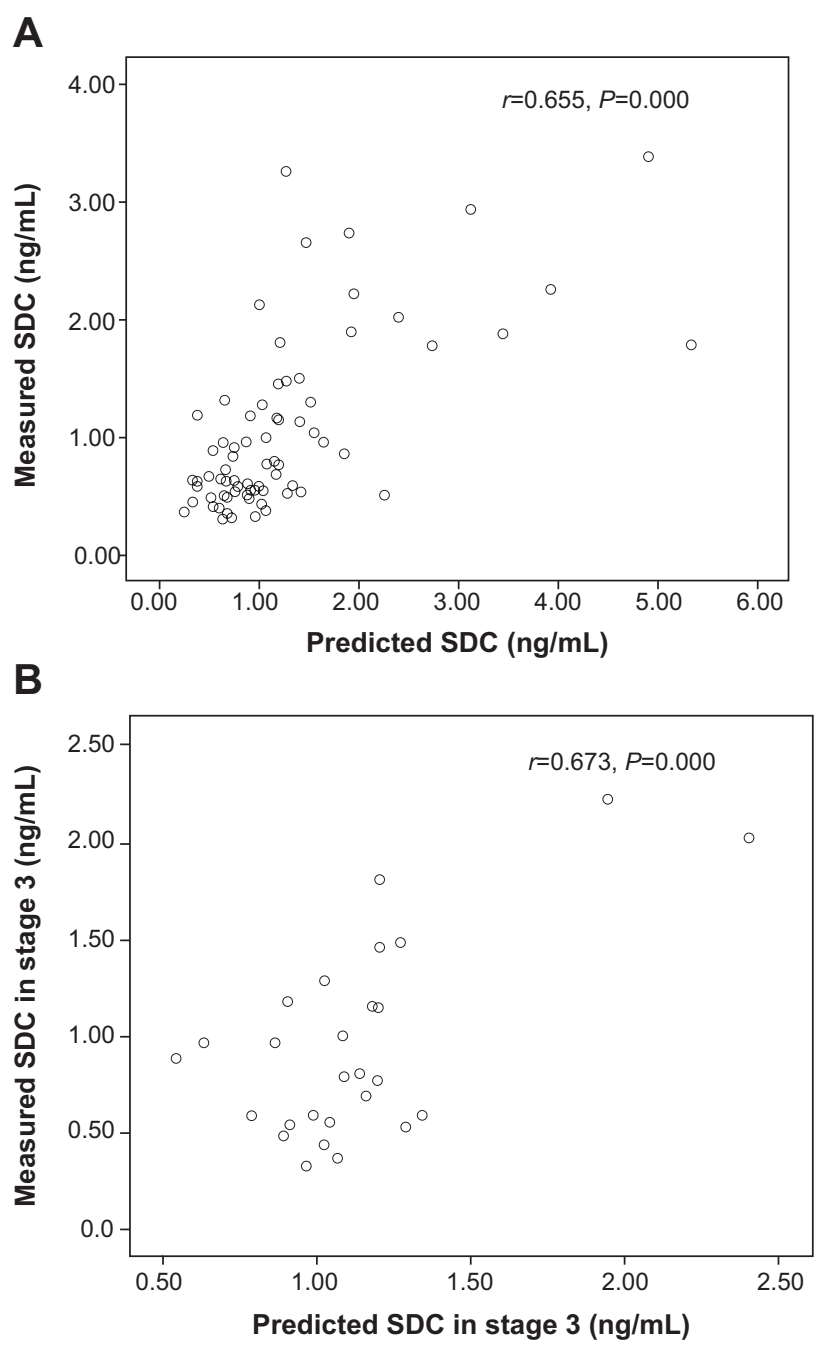

Figure 2 Correlation between measured and predicted SDC in all patients $(\mathbf{A})$ and in stage 3 patients (B).

Abbreviation: SDC, serum digoxin concentration.

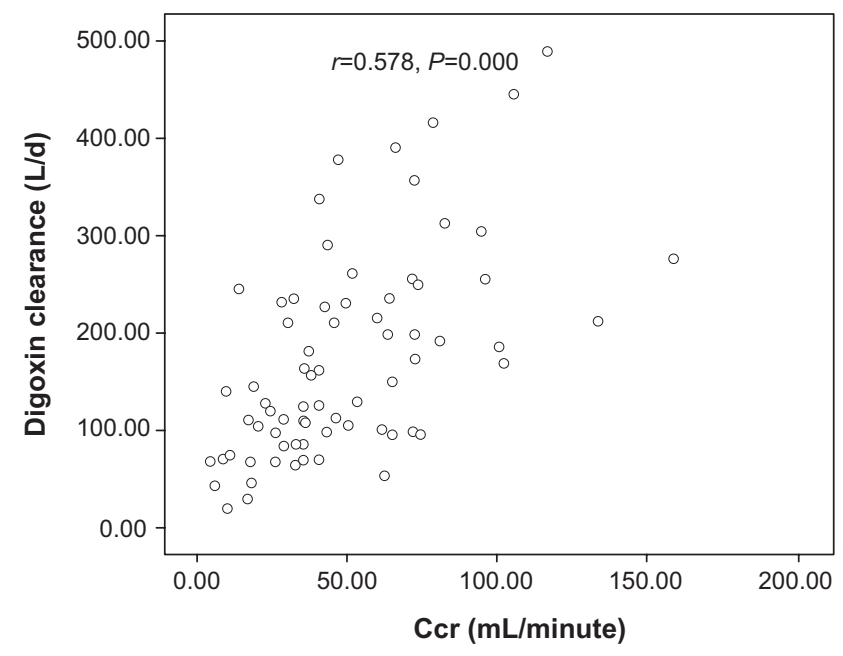

Figure 3 Correlation between clearance of digoxin and Ccr rate in all patients. Abbreviations: $\mathrm{Ccr}$, creatinine clearance rate; $d$, day. 
Table 3 Correlation between predicted and measured SDC, and correlation between CL and Ccr

\begin{tabular}{|c|c|c|c|c|}
\hline \multirow[t]{2}{*}{ Renal function stages } & \multicolumn{2}{|c|}{ Predicted and measured SDC } & \multicolumn{2}{|l|}{$\mathrm{CL}$ and $\mathrm{Ccr}$} \\
\hline & Correlation coefficient (r) & $P$-value & Correlation coefficient ( $r$ ) & $P$-value \\
\hline Stage I (n=9) & 0.2890 & 0.465 & -0.013 & 0.973 \\
\hline Stage $2(n=16)$ & -0.004 & 0.987 & 0.321 & 0.225 \\
\hline Stage $3(n=23)$ & 0.631 & $0.000^{*}$ & 0.320 & 0.103 \\
\hline Stage $4(n=13)$ & 0.492 & 0.088 & 0.422 & 0.517 \\
\hline Stage $5(n=7)$ & 0.082 & 0.862 & 0.572 & 0.180 \\
\hline Total $(\mathrm{n}=72)$ & 0.655 & $0.000^{*}$ & 0.578 & $0.000 *$ \\
\hline
\end{tabular}

Note: *Statistically significant.

Abbreviations: $\mathrm{Ccr}$, creatinine clearance rate; SDC, serum digoxin concentration; $\mathrm{CL}$, clearance.

These results are the same as those reported by Konishi ${ }^{5}$ and Muzzarelli ${ }^{14}$ in all inclusion patients. However, when considering the effect of renal function, the results of correlation between predicted and measured SDC were largely nonsignificant, only significant in stage 3 patients. Our results are similar to those reported by González-López and Tutor. ${ }^{15}$ The reason for these differences could lie in the different analytical methods used to determine digoxin levels. Morris et al reported positive interference from spironolactone in measurement of digoxin levels using the fluorescence polarization immunoassay marketed by Abbott Diagnostics for application on the TDxFLx ${ }^{\circledR}$ analyzer. ${ }^{16}$ Later, other investigators confirmed that spironolactone and canrenone interfered with the fluorescence polarization immunoassay as well as other immunoassays commonly used for digoxin, ${ }^{17-19}$ while the newer Abbott Architect i1000 immunology analyzer was free from interference by spironolactone..$^{20}$ Other reasons for the low predictability of the Konishi equation could be the variation in nonrenal digoxin CL in different populations, reduced volume of distribution, and reduced myocardial sensitivity. ${ }^{21}$ The results of our study cast doubt on the suitability of the Konishi equation for adjustment of drug dosage in clinical practice in Chinese patients, which could only be applied in stage 3 patients. As the limitation of our study, it will be necessary to further expand the number of patients in every stage of renal function in future studies to verify the original Konishi model in Chinese patients.

\section{Disclosure}

The authors report no conflicts of interest in this work.

\section{References}

1. Passmore AP, Johnston GD. Digoxin toxicity in the aged. Characterising and avoiding the problem. Drugs Aging. 1991;1(5):364-379.

2. Duhme D, Greenblatt D, Koch-Weser J. Reduction of digoxin toxicity associated with measurement of serum levels. A report from the Boston Collaborative Drug Surveillance Program. Ann Intern Med. 1974;80(4):516-519.
3. Gheorghiade M, Adams K, Colucci W. Digoxin in the management of cardiovascular disorders. Circulation. 2004;109(24):2959-2964.

4. Hunt SA, Abraham WT, Chin MH, et al. Focused update incorporated into the ACC/AHA 2005 Guidelines for the Diagnosis and Management of Heart Failure in Adults A Report of the American College of Cardiology Foundation/American Heart Association Task Force on Practice Guidelines Developed in Collaboration with the International Society for Heart and Lung Transplantation. J Am Coll Cardiol. 2009;53(15):e1-e90.

5. Konishi H, Shimizu S, Chiba M, Minouchi T, Koida M, Yamaji A. Predictive performance of serum digoxin concentration in patients with congestive heart failure by a hyper-bolic model based on creatinine clearance. J Clin Pharm Ther. 2002;27(4):257-265.

6. Cockcroft DW, Gault MH. Prediction of creatinine clearance from serum creatinine. Nephron. 1976;16(1):31-34.

7. Levey AS, Coresh J, Balk E, et al. National Kidney Foundation practice guidelines for chronic kidney disease: evaluation, classification, and stratification. Ann Intern Med. 2003;139(2):137-147.

8. Dobbs RJ, Nicholson PW, Denham MJ, Dobbs SM, O’Neill CJ. Therapeutic drug monitoring of digoxin: help or hindrance? Eur J Clin Pharmacol. 1986;31(4):491-495.

9. Pervaiz MH, Dickinson MG, Yamani M. Is digoxin a drug of the past? Cleve Clin J Med. 2006;73(9):821-824.

10. Vaz Pérez A, Otawa K, Zimmermann AV, et al. The impact of impaired renal function on mortality in patients with acutely decompensated chronic heart failure. Eur J Heart Fail. 2010;12(2):122-128.

11. Yancy CW, Jessup M, Bozkurt B, et al. 2013 ACCF/AHA Guideline for the Management of Heart Failure: A Report of the American College of Cardiology Foundation/American Heart Association Task Force on Practice Guidelines. J Am Coll Cardiol. 2013;62(16):e147-e239.

12. Mangoni AA, Jackson SH. Age-related changes in pharmacokinetics and pharmacodynamics: basic principles and practical applications. Br J Clin Pharmacol. 2004;57(1):6-14.

13. Mehvar R. Estimation of pharmacokinetic parameters based on the patient-adjusted population data. Am J Pharm Educ. 2006;70(5):96.

14. Muzzarelli S, Stricker H, Pfister O, Foglia P, Moschovitis G, Mombelli G. Individual dosage of digoxin in patients with heart failure. QJM. 2011;104(4):309-317.

15. González-López J, Tutor JC. Diagnostic accuracy of a hyperbolic model in predicting digoxin concentrations based on glomerular filtration rates. Nefrologia. 2011;31(5):573-578.

16. Morris RG, Frewin DB, Taylor WB, Glistak ML, Lehmann DR. The effect of renal and hepatic impairment and of spironolactone on serum digoxin assay. Eur J Clin Pharmacol. 1988;34(3):233-239.

17. Pleasants RA, Williams DM, Porter RS, Gadsden RH Sr. Reassessment of cross-reactivity of spironolactone metabolites with four digoxin assays. Ther Drug Monit. 1989;11(2):200-204.

18. Foukaridis GN. Influence of spironolactone and its metabolite canrenone on serum digoxin assays. Ther Drug Monit. 1990;12(1):82-84.

19. Stone JA, Soldin SJ. An update on digoxin. Clin Chem. 1989;35(7): $1326-1331$. 
20. DeFrance A, Armbruster D, Petty D, Cooper KC, Dasgupta A. Abbott Architect clinical chemistry and immunoassay systems: digoxin assays are free of interferences from spironolactone, potassium canrenoate, and their common metabolite canrenone. Ther Drug Monit. 2011;33(1): $128-131$.
21. Kramer P. Digitalis pharmacokinetics and therapy with respect to impaired renal function. Klin Wochenschr. 1977;55(1):1-11.

\section{Publish your work in this journal}

Clinical Interventions in Aging is an international, peer-reviewed journal focusing on evidence-based reports on the value or lack thereof of treatments intended to prevent or delay the onset of maladaptive correlates of aging in human beings. This journal is indexed on PubMed Central, MedLine,
CAS, Scopus and the Elsevier Bibliographic databases. The manuscript management system is completely online and includes a very quick and fair peer-review system, which is all easy to use. Visit http://www.dovepress. com/testimonials.php to read real quotes from published authors. 